Supporting Information

\title{
Ternary Amorphous Solid Dispersions Containing a High Viscosity Polymer and Mesoporous Silica Enhance Dissolution Performance.
}

\author{
Masataka Hanada ${ }^{1,2}$, Scott V. Jermain ${ }^{1,3}$, Stephen A. Thompson ${ }^{1}$, Hirosuke Furuta ${ }^{2}$, \\ Mamoru Fukuda ${ }^{2}$, Robert O. Williams III ${ }^{1}$ \\ 1: Division of Molecular Pharmaceutics and Drug Delivery, College of Pharmacy, The University of Texas at \\ Austin, 2409 University Ave., A1920, Austin, TX 78712, USA \\ 2: CMC Research Laboratory, Watarase Research Center, Kyorin Pharmaceutical Co., Ltd., 1848 Nogi, Nogi-machi, \\ Shimotsuga-gun, Tochigi, 329-0114, Japan \\ 3: Formulation and Process Development, Gilead Sciences Inc., 333 Lakeside Dr., Foster City, CA 94404, USA
}

\section{【Methods for Figure S1, S2 and S3】}

\section{Polarized light microscopy (PLM)}

Each ITZ, ITZ/AF15 (5:2, w/w) and ITZ/AF4M (5:2, w/w) was dissolved in DMSO at $5 \mathrm{mg} / \mathrm{mL}$ and prepared a total volume of $1 \mathrm{~mL}$, respectively. The $200 \mu \mathrm{L}$ of stock solution was mixed with $800 \mu \mathrm{L}$ of neutral media prepared from $0.1 \mathrm{~N} \mathrm{HCl} / 0.2 \mathrm{M} \mathrm{Na}_{3} \mathrm{PO}_{4}(750: 250$, v/v). Samples were prepared at each sampling points: $0,240 \mathrm{~min}$. The samples were shaken at a speed of $250 \mathrm{rpm}$ and temperature of $37^{\circ} \mathrm{C}$ by thermo-shaker (MSC-100, Bio Medical Science, Tokyo, Japan), and centrifuged at 12,000 rpm for 3 min. The $20 \mu \mathrm{L}$ of the above precipitation samples were put on slide glass with cover glass and observed by using cross-Polarized light microscopy (PLM). The sample images were captured by Nikon Eclipse LV100 POL microscope with 10x magnification equipped with DS-Fi3 digital camera and a $\lambda(530 \mathrm{~nm})$ tint plate (Nikon Co., Tokyo, Japan). 

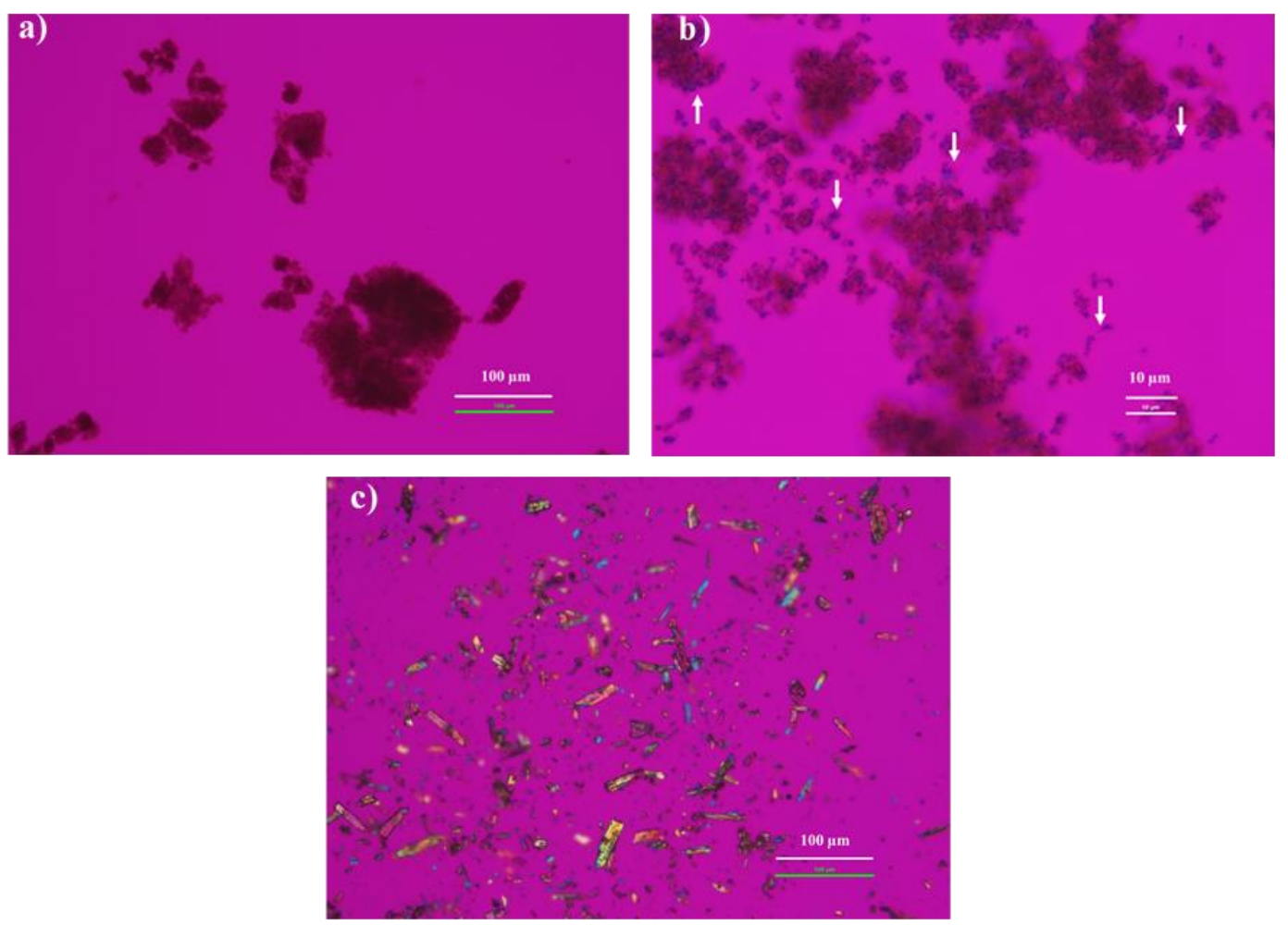

Figure S1. PLM of a) ITZ precipitation (time point: $0 \mathrm{~min}$ ) at $200 \times$ magnification and b) ITZ precipitation (time point: $240 \mathrm{~min}$ ) at 1,000× magnification and c) ITZ crystal powder at 200× magnification. White arrow indicates representative ITZ crystal. 

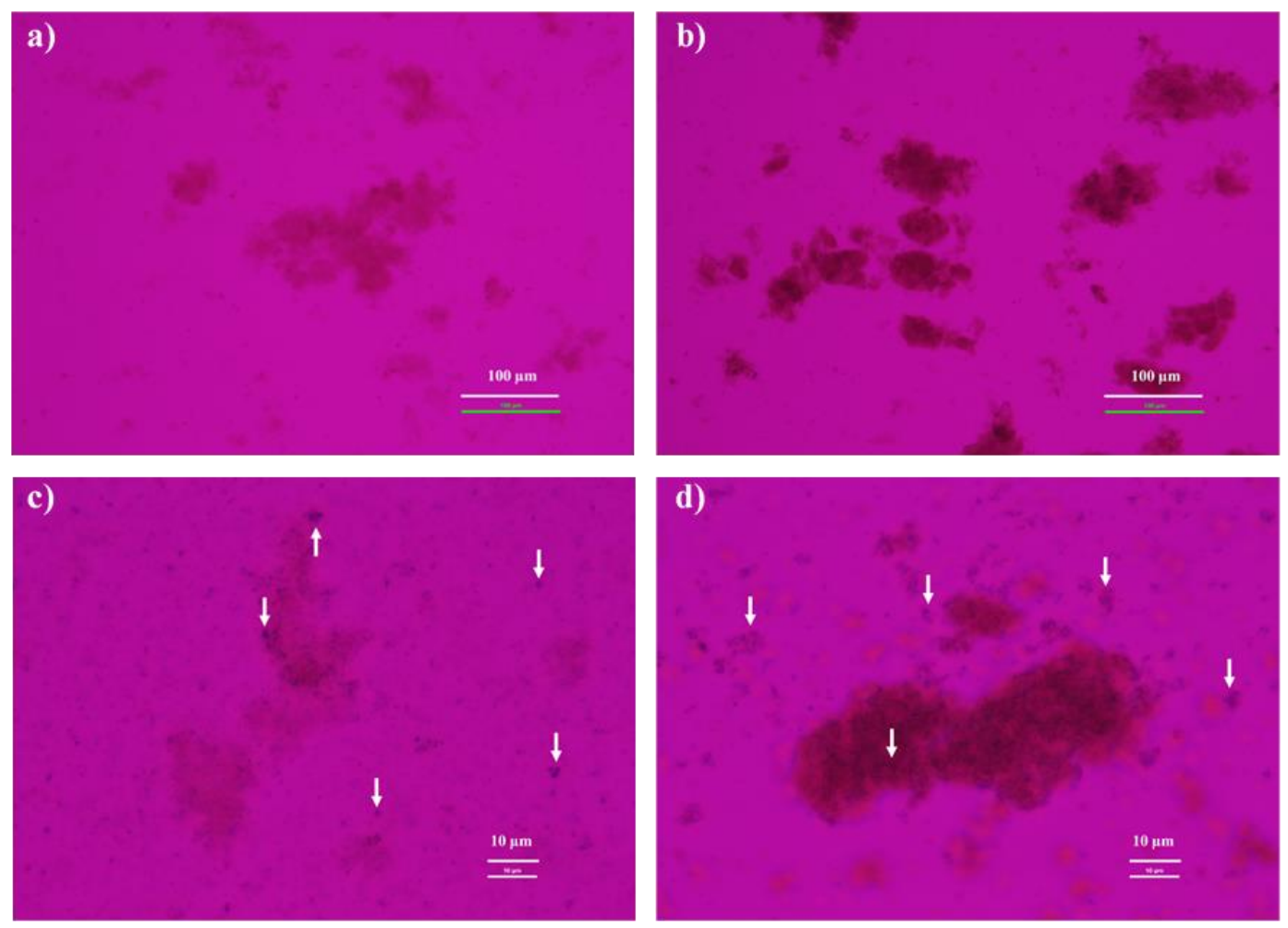

Figure S2. PLM of ITZ/AF polymer precipitation. a) ITZ:AF4M precipitation (time point: 0 min) at 200× magnification, b) ITZ:AF15 precipitation (time point: $0 \mathrm{~min}$ ) at 200× magnification, c) ITZ:AF4M precipitation (time point: $240 \mathrm{~min}$ ) at 1,000× magnification, d) ITZ:AF15 precipitation (time point: 240 min) at 1,000× magnification. White arrow indicates representative ITZ crystal.

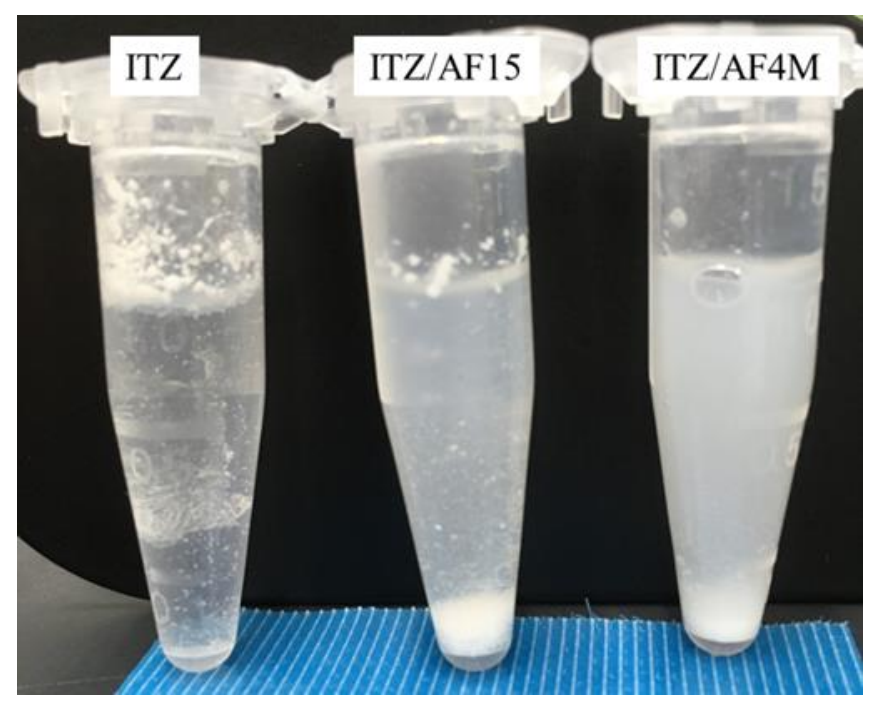

Figure S3. Appearance of ITZ and ITZ/AF polymer precipitations at $240 \mathrm{~min}$ after centrifugation. 
Table S1. Particle size of ternary ASD granules containing different AF grades in $\mathrm{pH}$ transition test. ITZ concentration of each sample was $60 \mu \mathrm{g} / \mathrm{mL}$, and testing was performed in $0.1 \mathrm{~N} \mathrm{HCl}$ followed by $\mathrm{pH}$ adjustment to 6.8 with $0.2 \mathrm{M} \mathrm{Na}_{3} \mathrm{PO}_{4}$ solution at $37{ }^{\circ} \mathrm{C}$.

\begin{tabular}{|c|c|c|c|c|c|}
\hline \multicolumn{2}{|c|}{ Time point (min) } & 5 & 30 & 60 & 120 \\
\hline & & Particle size $(\mathrm{nm})$ & Particle size $(\mathrm{nm})$ & Particle size $(\mathrm{nm})$ & Particle size $(\mathrm{nm})$ \\
\hline \multirow{3}{*}{ ITZ/AF4M } & $\mathrm{n}=1$ & 264.9 & 364.1 & N.D. & N.D. \\
\hline & $\mathrm{n}=2$ & 307.7 & 395.6 & N.D. & N.D. \\
\hline & $n=3$ & 324.0 & 343.9 & N.D. & N.D. \\
\hline \multicolumn{2}{|c|}{ Average } & 298.9 & 367.9 & - & - \\
\hline \multicolumn{2}{|c|}{ S.D. } & 30.5 & 26.1 & - & - \\
\hline \multicolumn{2}{|c|}{ Time point $(\mathrm{min})$} & 5 & 30 & 60 & 120 \\
\hline & & Particle size $(\mathrm{nm})$ & Particle size $(\mathrm{nm})$ & Particle size $(\mathrm{nm})$ & Particle size $(\mathrm{nm})$ \\
\hline \multirow{3}{*}{ ITZ/AF15 } & $\mathrm{n}=1$ & N.D. & N.D. & N.D. & N.D. \\
\hline & $\mathrm{n}=2$ & N.D. & N.D. & N.D. & N.D. \\
\hline & $\mathrm{n}=3$ & N.D. & N.D. & N.D. & N.D. \\
\hline \multicolumn{2}{|c|}{ Average } & - & - & - & - \\
\hline \multicolumn{2}{|c|}{ S.D. } & - & - & - & - \\
\hline
\end{tabular}

N.D.: Not detected 


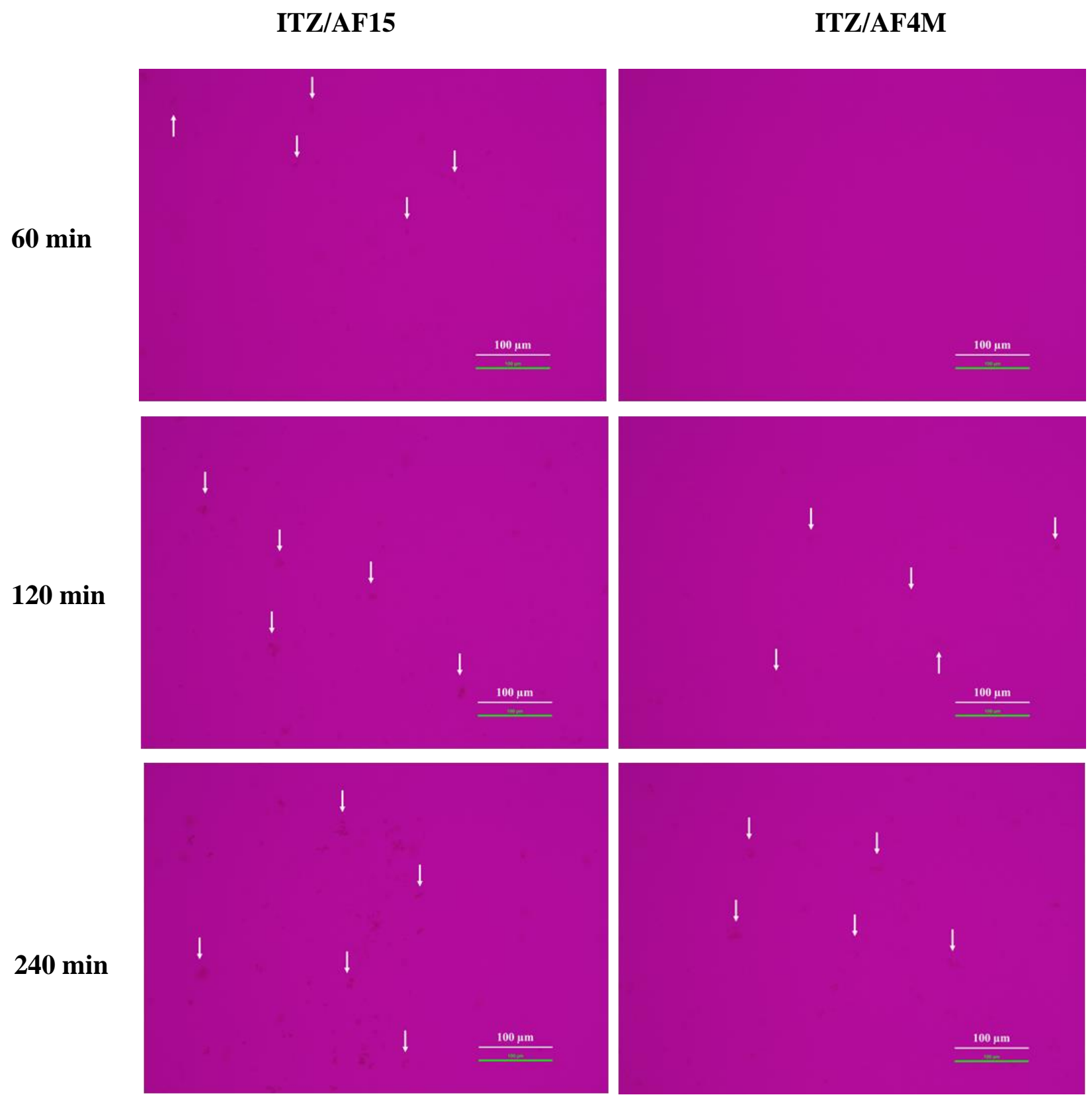

Figure S4. PLM of ITZ/AF polymer precipitation at 200× magnification. White arrow indicates representative amorphous precipitation. 
a)

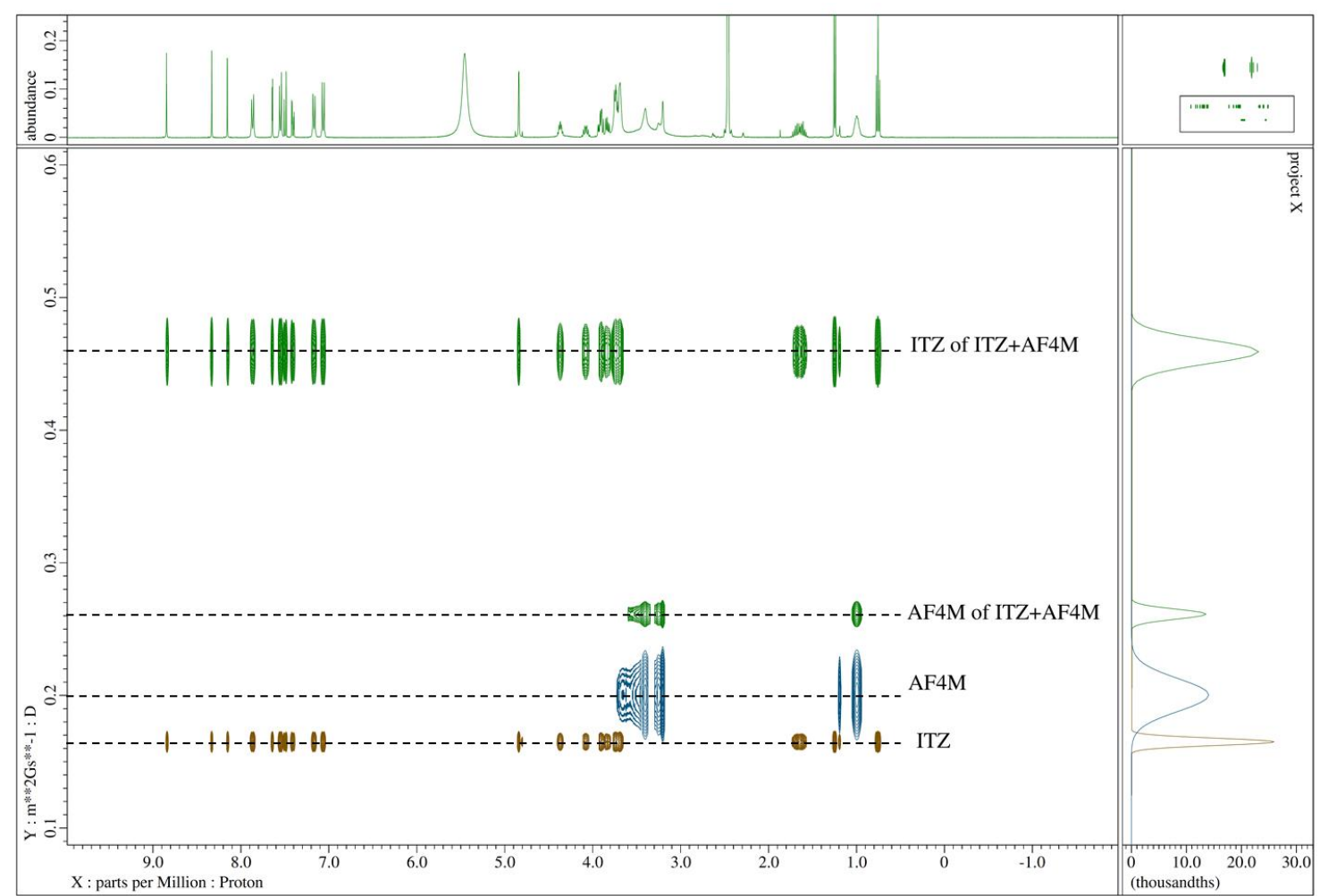

b)

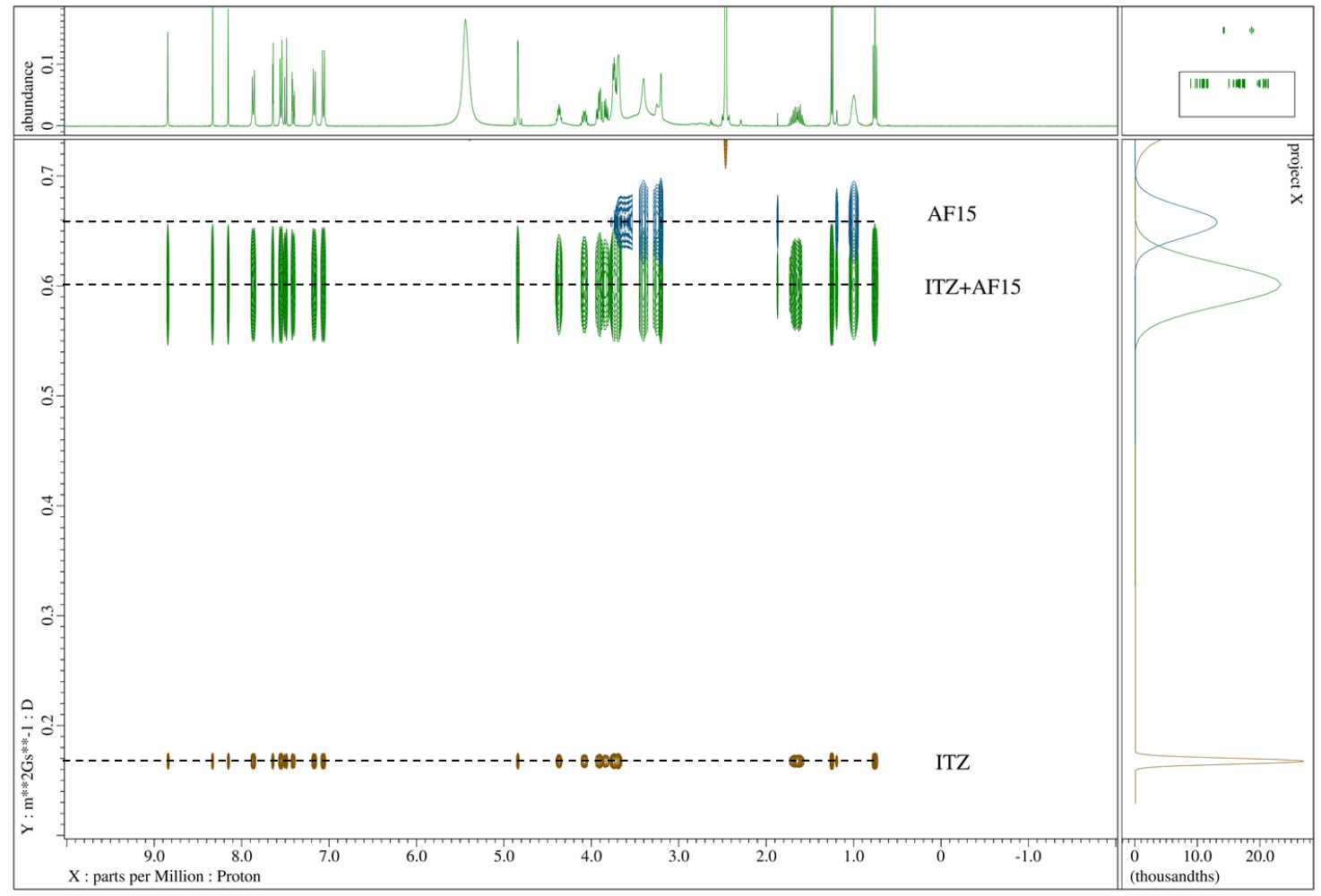

Figure S5. Investigation of solution state interaction by DOSY. a) DOSY spectra of ITZ:AF4M (5:2, w/w, green), ITZ (brown), and AF4M (blue); b) DOSY spectra of ITZ:AF15 (5:2, w/w, green), ITZ (brown), and $\mathrm{AF} 15$ (blue) in $0.1 \mathrm{~N} \mathrm{DCl} / \mathrm{DMSO}-\mathrm{d}_{6}$. 


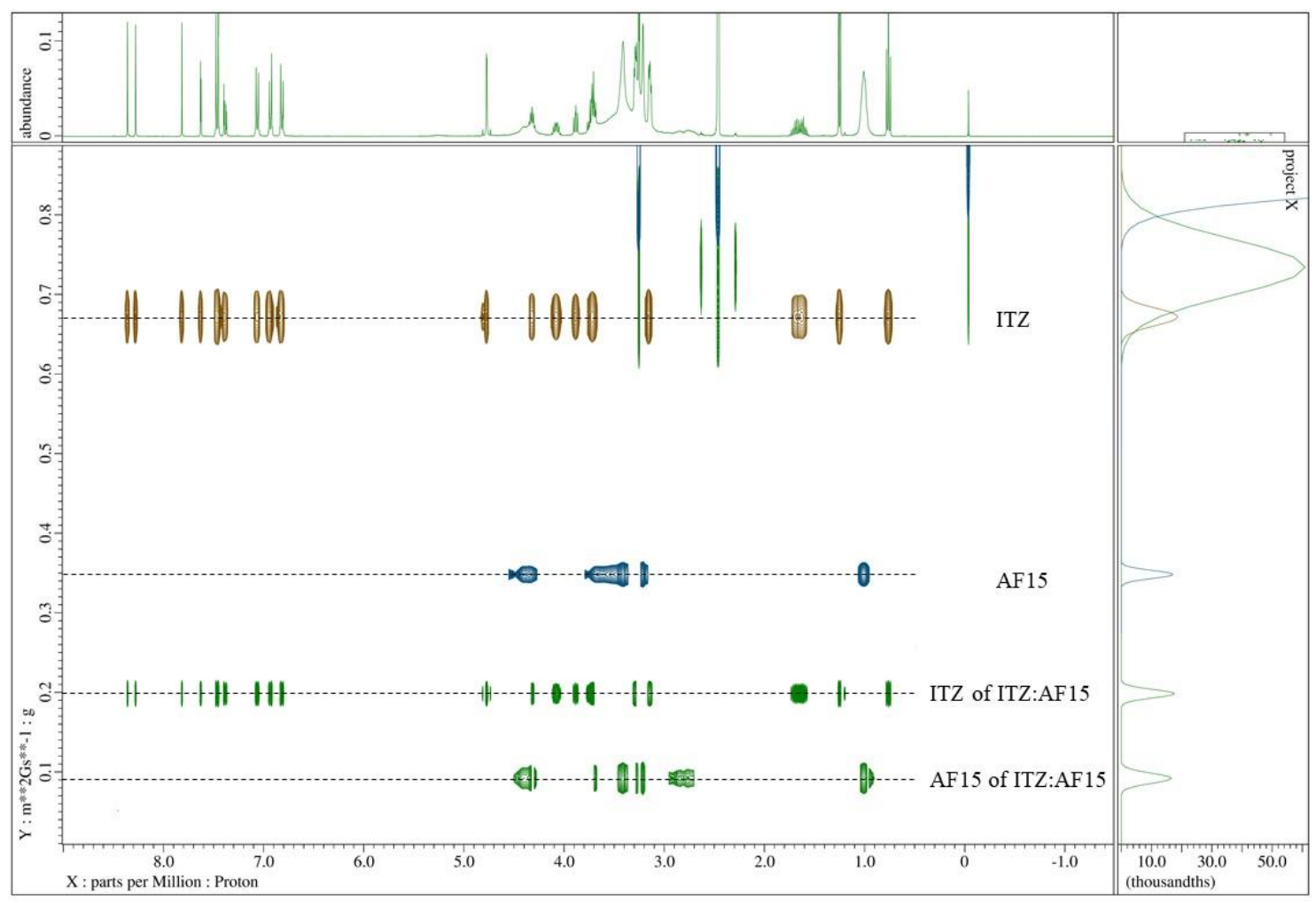

Figure S6. Investigation of solution state interaction by DOSY. DOSY spectra of ITZ:AF15 (5:5, w/w, green), ITZ (brown), and AF15 (blue) in DMSO-d $\mathrm{d}_{6}$. 


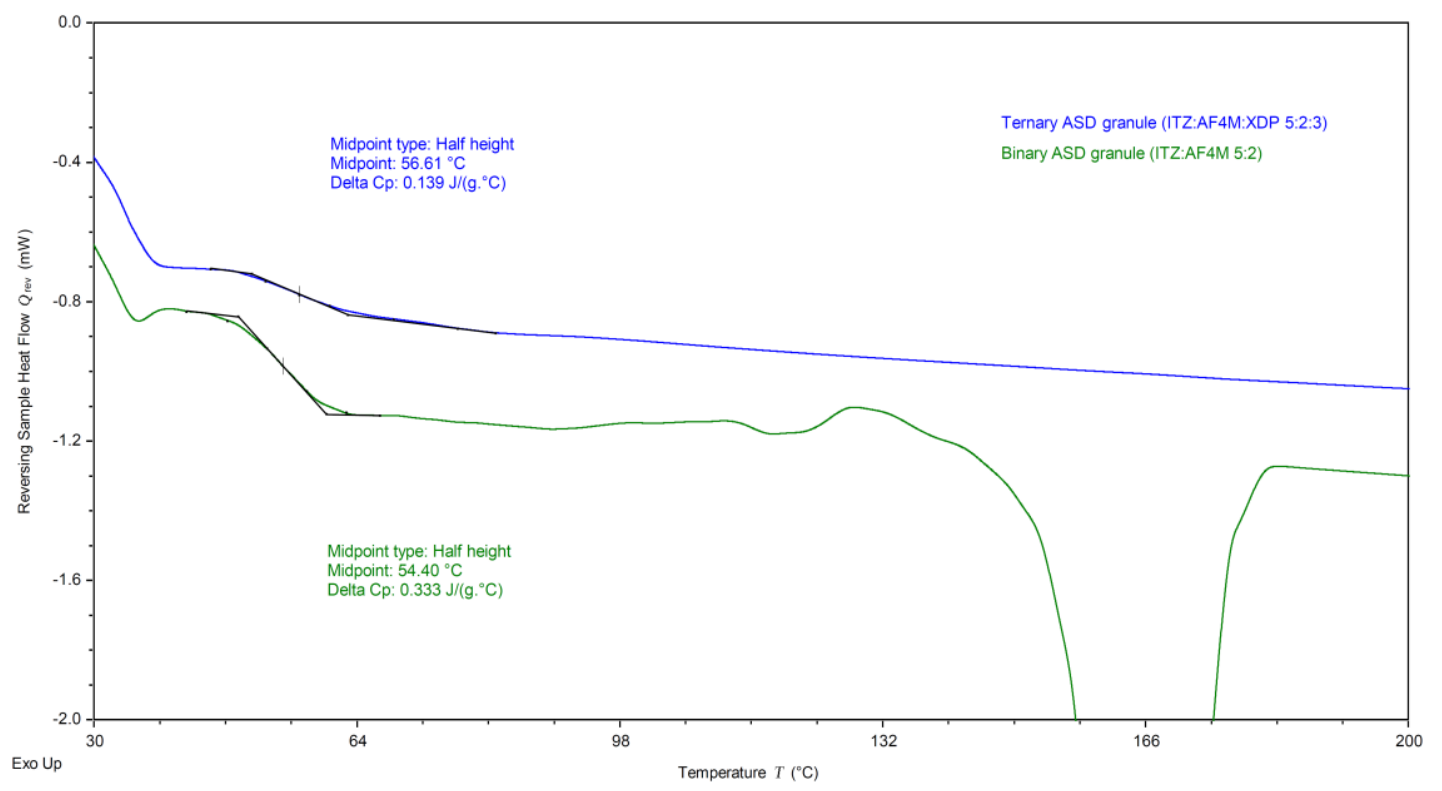

TA Instruments Trios V4.5.1.42498

Figure S7. $\mathrm{T}_{\mathrm{g}}$ of ternary ASD (ITZ:AF4M:XDP = 5:2:3, w/w) prepared by HME and binary ASD (ITZ:AF4M = 5:2, w/w) prepared by MQ.

Table S2 $\mathrm{T}_{\mathrm{g}}$ and heat capacity $(\triangle \mathrm{Cp})$ with different ratios of ITZ/AF4M and XDP.

\begin{tabular}{|c|c|c|c|c|c|c|}
\hline ITZ/AF4M ratio (\%) & \multicolumn{2}{|c|}{50} & \multicolumn{2}{|c|}{60} & \multicolumn{2}{|c|}{70} \\
\hline XDP ratio $(\%)$ & \multicolumn{2}{|c|}{50} & \multicolumn{2}{|c|}{40} & \multicolumn{2}{|c|}{30} \\
\hline & $\mathrm{T}_{\mathrm{g}}\left({ }^{\circ} \mathrm{C}\right)$ & $\Delta \mathrm{Cp}\left(\mathrm{J} /\left(\mathrm{g} \cdot{ }^{\circ} \mathrm{C}\right)\right.$ & $\mathrm{T}_{\mathrm{g}}\left({ }^{\circ} \mathrm{C}\right)$ & $\Delta \mathrm{Cp}\left(\mathrm{J} /\left(\mathrm{g} \cdot{ }^{\circ} \mathrm{C}\right)\right.$ & $\mathrm{T}_{\mathrm{g}}\left({ }^{\circ} \mathrm{C}\right)$ & $\Delta \mathrm{Cp}\left(\mathrm{J} /\left(\mathrm{g} \cdot{ }^{\circ} \mathrm{C}\right)\right.$ \\
\hline $\mathrm{n}=1$ & 59.14 & 0.076 & 58.62 & 0.100 & 58.56 & 0.150 \\
\hline $\mathrm{n}=2$ & 59.07 & 0.070 & 59.04 & 0.097 & 58.48 & 0.159 \\
\hline$n=3$ & 59.11 & 0.062 & 58.76 & 0.098 & 58.46 & 0.141 \\
\hline Average & 59.11 & 0.069 & 58.81 & 0.098 & 58.50 & 0.150 \\
\hline S.D. & 0.04 & 0.007 & 0.21 & 0.002 & 0.05 & 0.009 \\
\hline ITZ/AF4M ratio $(\%)$ & \multicolumn{2}{|c|}{80} & \multicolumn{2}{|c|}{90} & \multicolumn{2}{|c|}{100} \\
\hline XDP ratio (\%) & \multicolumn{2}{|c|}{20} & \multicolumn{2}{|c|}{10} & \multicolumn{2}{|c|}{0} \\
\hline & $\mathrm{T}_{\mathrm{g}}\left({ }^{\circ} \mathrm{C}\right)$ & $\Delta \mathrm{Cp}\left(\mathrm{J} /\left(\mathrm{g} \cdot{ }^{\circ} \mathrm{C}\right)\right.$ & $\mathrm{T}_{\mathrm{g}}\left({ }^{\circ} \mathrm{C}\right)$ & $\Delta \mathrm{Cp}\left(\mathrm{J} /\left(\mathrm{g} \cdot{ }^{\circ} \mathrm{C}\right)\right.$ & $\mathrm{T}_{\mathrm{g}}\left({ }^{\circ} \mathrm{C}\right)$ & $\Delta \mathrm{Cp}\left(\mathrm{J} /\left(\mathrm{g} \cdot{ }^{\circ} \mathrm{C}\right)\right.$ \\
\hline $\mathrm{n}=1$ & 59.30 & 0.205 & 59.50 & 0.323 & 59.02 & 0.386 \\
\hline $\mathrm{n}=2$ & 58.92 & 0.211 & 59.85 & 0.313 & 58.84 & 0.389 \\
\hline $\mathrm{n}=3$ & 58.95 & 0.210 & 59.68 & 0.315 & 59.06 & 0.383 \\
\hline Average & 59.06 & 0.209 & 59.68 & 0.317 & 58.97 & 0.386 \\
\hline S.D. & 0.21 & 0.003 & 0.18 & 0.005 & 0.12 & 0.003 \\
\hline
\end{tabular}




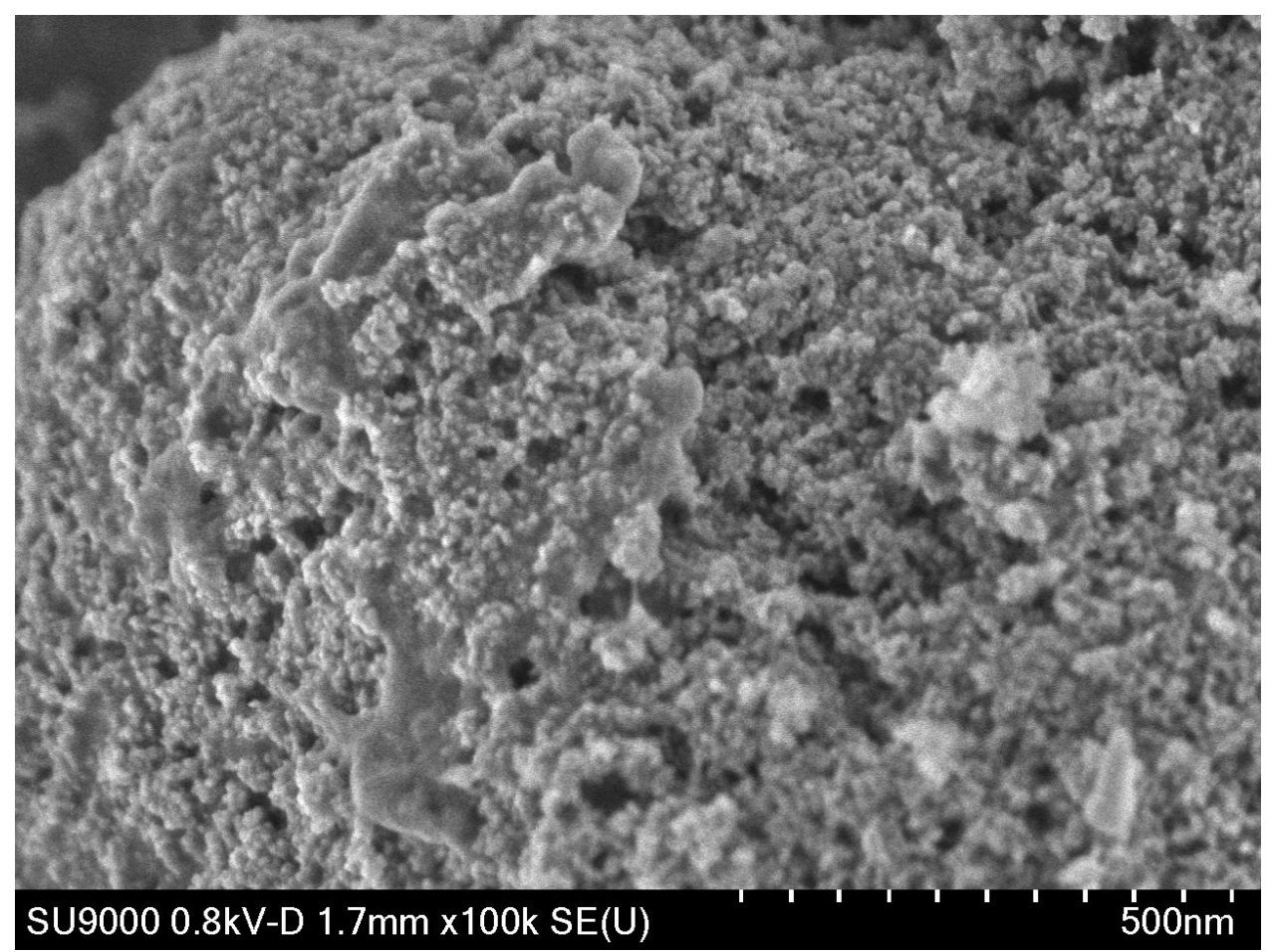

Figure S8. SEM image of a ternary ASD (ITZ:AF4M:XDP) granule surface $(100,000 \times$ magnification). 


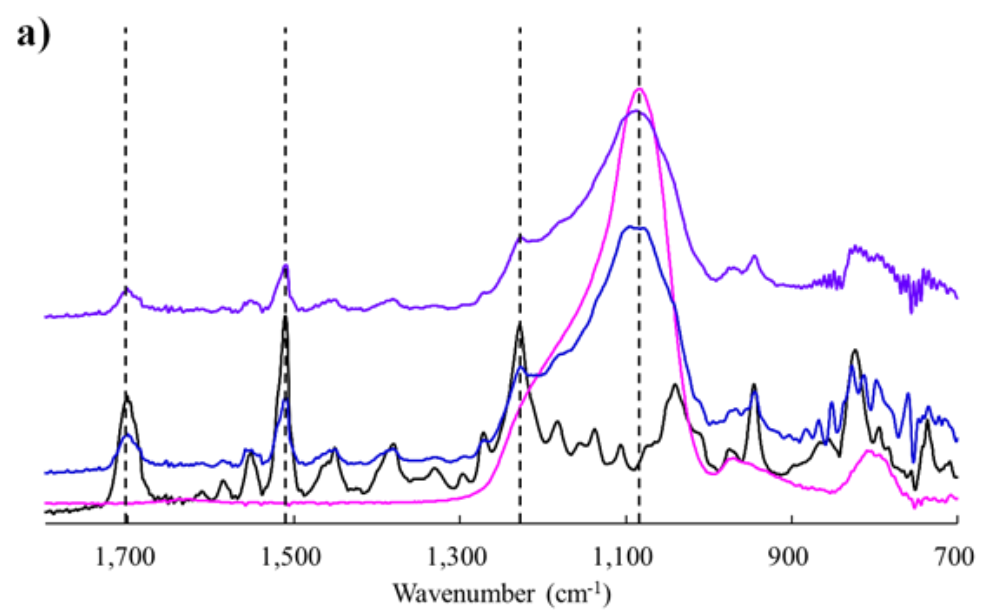

—Amorphous ITZ —XDP -ITZ:XDP (5:3) -ITZ:AF4M:XDP (5:2:3)

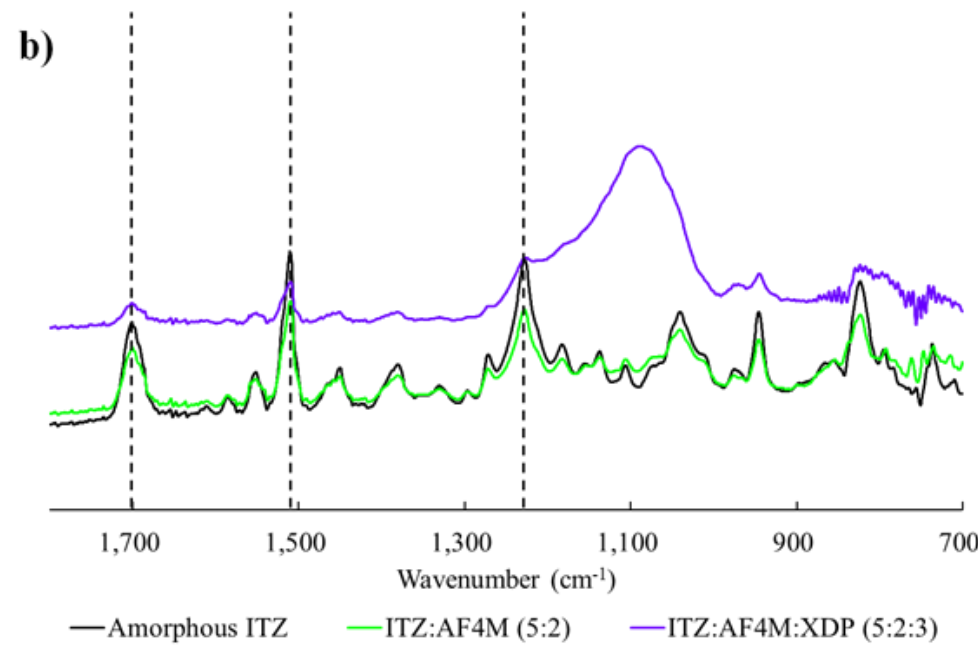

Figure S9. Investigation of solid state interaction in ternary ASD granules compared with binary ASD by FTIR. a) FTIR spectra $\left(700-1800 \mathrm{~cm}^{-1}\right)$ of ternary ASD granules and binary ASD (ITZ:XDP); b) FTIR spectra $\left(700-1800 \mathrm{~cm}^{-1}\right)$ of ternary ASD granules and binary ASD (ITZ:AF4M). 